\title{
Cross-leg repair of large soft-tissue defects in distal sites of the feet by distally based neuro- fasciocutaneous flaps with perforating vessels
}

\author{
Z.Q. Wang, Y.L. Cao, Y.F. Huang, D.Q. Liu and X.F. Li \\ Department of Plastic and Reconstructive Surgery, \\ The Third Hospital of Liao-Ning Medical College, Jinzhou, China \\ Corresponding author: Z.Q. Wang \\ E-mail: zqxden@yeah.net \\ Genet. Mol. Res. 13 (3): 5484-5491 (2014) \\ Received June 3, 2013 \\ Accepted October 4, 2013 \\ Published July 25, 2014 \\ DOI http://dx.doi.org/10.4238/2014.July.25.1
}

\begin{abstract}
The objective of this study was to introduce a method for repairing large soft-tissue defects on the foot. Distally based neurofasciocutaneous flaps with perforating vessels were designed along the saphenous and sural neurovascular axes. The cutaneous perforating branches of the major arteries of the lower extremities were used as pedicles, which provided a rotation arc for the cross-leg flap to cover the large-sized soft-tissue defects on the foot. We transferred 6 neurocutaneous vascular axial flaps, including 4 saphenous neurocutaneous axial flaps (ranging from $25 \times 13$ to $17 \times 9 \mathrm{~cm}$ in area) with posterior tibial perforators as the pedicle, and 2 sural neurocutaneous axial flaps (ranging from $29 \times 12$ to $18 \times 7 \mathrm{~cm}$ in area) supplied by the perforating branches of the peroneal vessels. These 6 cases of neuro-fasciocutaneous flaps survived with satisfactory cosmetic appearances and functional results on follow-up at 8 to 17 months post-surgery. Placing a distally based neurofasciocutaneous cross-leg flap with perforating vessels is an effective method for repairing large-sized soft-tissue defects on the foot.
\end{abstract}

Key words: Perforator vessels; Neurocutaneous axial flap; Soft-tissue defect; Foot 


\section{INTRODUCTION}

In clinical practice, foot injuries are simultaneously accompanied by difficult to repair soft-tissue defects because of several factors. First, the foot is located at the distal aspect of the lower limb and its degree of active movement is limited compared to the hand, for which orthopedic procedures can be easily performed and soft-tissue defects can be repaired with flaps transferred from the trunk. Second, the subcutaneous adipose- and soft-tissue layers of the foot are thin, such that when local injury sets in, the deep tissues of the lower extremities, including the bones, blood vessels, tendons, nerves, or other important anatomical parts, may be inadvertently exposed. Therefore, when soft-tissue defects cover a large body surface area, these defects must be repaired with large flaps. Lastly, the elasticity of the skin and subcutaneous tissue of the foot is limited, especially on the plantar surface of the foot. Thus, only smaller local flaps can be used to repair local defects. The repair of a large-sized soft-tissue defect on the foot using a reverse flap transfer on the same area near the flap is a convenient and economical method.

If there is a large-sized soft-tissue defect on the foot with exposed deep tissues, repair using a reverse flap transfer on the ipsilateral leg cannot be completed in this precarious location because the vascular pedicle blood flow must be maintained. In this situation, free flap transposition is an effective method of repairing soft-tissue defects of the foot. However, in selected cases, such as in electrical burns that result in damage to the posterior tibial artery, which is one of the main arteries of the lower extremities, difficulties will be encountered when free flaps are used.

Based on the above scenarios that are encountered in clinical practice, this study reports on the development of a surgical procedure by which the contralateral leg is used to form larger reverse flaps to repair extensive foot wound injuries. The main requirements for a flap planning are as follows: the flap must be of sufficient area and length to extend to and cover the soft-tissue defect on the foot; a flap must have adequate blood supply and blood pressure to satisfy these two conditions; and formation of the flap pedicle must be as close as possible to the soft-tissue defect on the distal foot area concerned. To accomplish these goals, the terminal perforating branch of the posterior tibial and peroneal arteries must be considered as the flap rotation axis, and the distally located flap pedicle must include the cutaneous nerve, accompanying nutrient vessels, and the deep fascia. The use of distally based neuro-fasciocutaneous flaps with perforating vessels is an ideal method for conducting cross-leg flap transfer to repair a large-sized soft-tissue defect on the foot.

In this study, this surgical procedure was clinically performed with 6 successful cases.

\section{MATERIAL AND METHODS}

\section{General patient data}

Our study cohort included 6 patients ( 5 men and 1 woman). Patient ages ranged from 21 to 45 years, and the median age was 32 years. All cases involved electrical burns. The physical dimensions of the soft-tissue defects in our patients were as follows: from 7 × 6 to 13 $\mathrm{x} 11 \mathrm{~cm}$. The physical dimensions of the posterior tibial artery perforator flap (with a pedicle consisting of the posterior tibial artery, saphenous nerve, nutrient vessels, and deep fascia) were as follows: from $25 \times 13$ to $17 \times 9 \mathrm{~cm}$. The physical dimensions of the peroneal artery perforator flap (with a pedicle consisting of the peroneal artery, sural nerve, nutrient vessels, and deep fascia) were as follows: from $29 \times 12$ to $18 \times 7 \mathrm{~cm}$. 


\section{Surgical technique}

Doppler ultrasound was used to determine the starting point of the perforating branches of the posterior tibial and peroneal arteries on the medial and on the lateral malleoli, respectively. The starting point was utilized as the flap rotation axis, which uses the accompanying cutaneous nerve as the flap axis. The surface projection of the sural nerve and the small saphenous vein is from the midpoint of the popliteal fossa to the interval between the lateral border and the midpoint of the Achilles tendon. The surface projection of the saphenous nerve and the great saphenous vein is from the medial condyle to the femur, medial to the tibia, proximal to the tip of the medial malleolus, and to the midpoint of the Achilles tendon. Based on the location and size of the foot wound, the terminal perforator of the posterior tibial artery or the peroneal artery is selected as the flap rotation axis point, at which point the accompanying cutaneous nerve, nutrient vessels, and deep fascia of the distal flap pedicle are preserved. The length and width of the flap should be $2-3 \mathrm{~cm}$ larger than the wound edge when the flaps are designed. The dissection of the flap starts distally from the flap. The flap is lifted up to the bottom of the deep fascia, which gradually separates the flap. The flap is then lifted towards the pedicle pivot point and precautions to keep the deep fascia intact during the course of the separation were observed. The wound edge of the deep fascia and skin was sutured, which can prevent the deep fascia from separating from the subcutaneous tissue. The diameter of the pedicle was kept at $5 \mathrm{~cm}$, which includes the cutaneous nerve, subcutaneous adipose tissue, and deep fascia. The veins were carefully isolated, and all hardened and engorged veins in the flap pedicle were ligated. The flaps were reversely transferred for suturing and covered the soft-tissue defect on the foot. The flap pedicle was covered with multiple layers of Vaseline-treated gauze bandages and the lower limb was stabilized in proper anatomical positions.

\section{RESULTS}

All 6 cases of distally based pediculated neuro-fasciocutaneous flaps with vascular perforators survived. During the follow-up period at 8-17 months post-surgery, the flap quality was excellent, and the recovery of cosmetic appearance and anatomical functions of the involved lower limbs were in all cases satisfactory.

A typical case is described as follows: Chen is a 25 -year-old male who sustained electrical burns on his hands and right lower limb. He was admitted to the hospital for 7 days because he was exposed to a $60,000-\mathrm{V}$ high-voltage electric wire. The physical examination on admission was as follows: his general physical condition and appearance were good, and his vital signs are stable. The patient had a $5 \times 3 \mathrm{~cm}$ necrotic cutaneous and soft-tissue defect on the web of skin between the first and second fingers, and on the thenar surface of the left hand. A necrotic soft-tissue defect was observed on the right palmar surface. A necrotic cavity having an area of $7 \times 4 \mathrm{~cm}$ on the back of the right foot ankle was also noted. The Achilles tendon and calcaneus were exposed, and the presence of pus was observed. The dorsal tibial arterial pulsation of the right foot disappeared. Dry necrotic defects were observed on the right second digit, the lateral aspect of the first digit, the soft tissues on the plantar surface of the right foot, and some bone parts. The interphalangeal and metatarsophalangeal joint cavities were exposed. Necrosis was also observed on the inner aspect of the third digit, in the soft tissue of the plantar surface, and some bone parts of the right foot, which had the interphalan- 
geal joints exposed. Almost a third of the soft tissue in the plantar aspect of the right foot had liquefaction necrosis, from the inside of the medial aspect and midline of the first digit to the lateral aspect and below the fifth digit, and up to $4 \mathrm{~cm}$ proximal on the right foot. Local flaps were placed on the soft-tissue defects on the dorsum, proximal, and plantar aspects of the right foot. After wound debridement, a $12 \times 10-\mathrm{cm}$ open wound defect was formed with the following parts of the right foot exposed: bone, tendon, interphalangeal joints of the first and third digits, metatarsophalangeal joints, and parts of the metatarsus.

An interphalangeal joint fusion (arthrodesis) using Kirschner fixation was performed. A posterior tibial artery perforator/neuro-fasciocutaneous flap with an intact saphenous nerve, nutrient vessels, and greater saphenous vein in the pedicle was transferred from the contralateral leg. The flap area was $25 \times 13 \mathrm{~cm}$. Cross-leg flap transfer was performed to repair cutaneous and soft-tissue defects on the proximal aspect of the right foot. Pedicle division was performed 3 weeks post-surgery. The surgical wounds were in the first stage of wound healing, but the sutured wounds on the dorsal aspect of the ankle underwent further organization because of the liquefaction necrosis. The wounds would heal after a series of dressings, and two additional surgeries were performed within a month from the first surgery. During the follow-up of this patient at 17 months post-surgery, the appearance and general contour of the affected foot were good and the patient could continuously ambulate it for more than $8 \mathrm{~h}$. The flaps are not ulcerated, as shown in Figures 1 to 4.
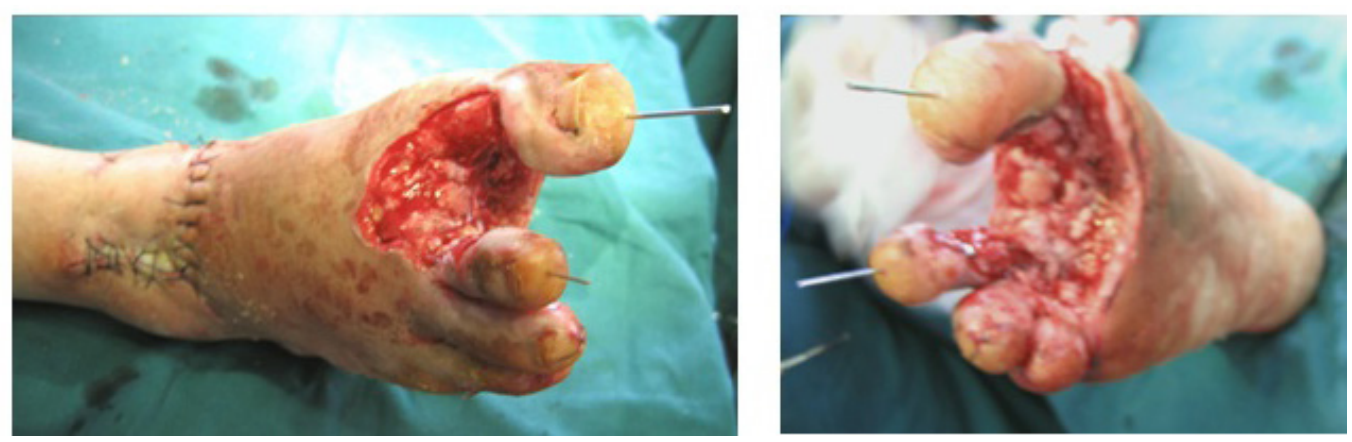

Figure 1. Electrically burned wounds in the right foot after debridement.
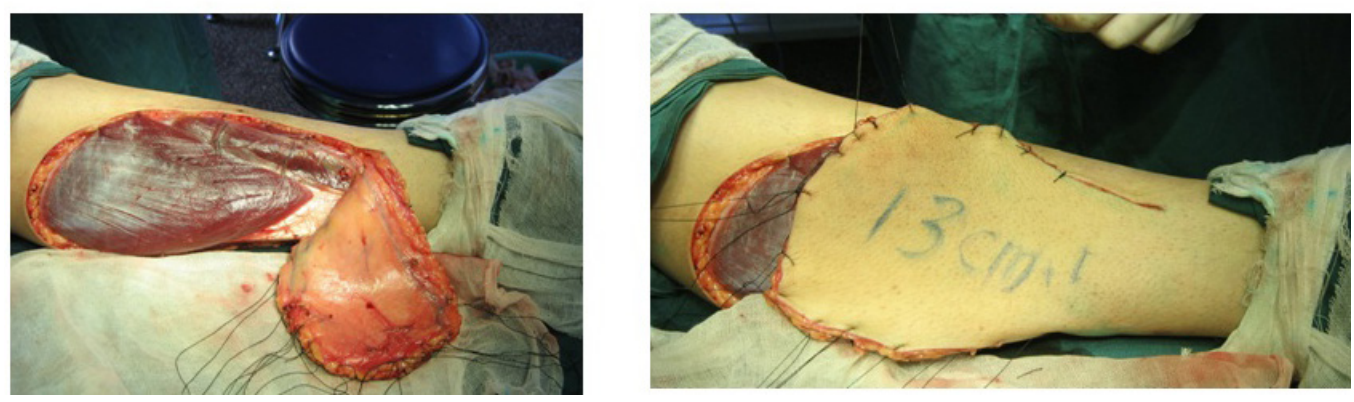

Figure 2. Neurocutaneous flap in the contralateral leg based on a posterior tibial artery perforator. 

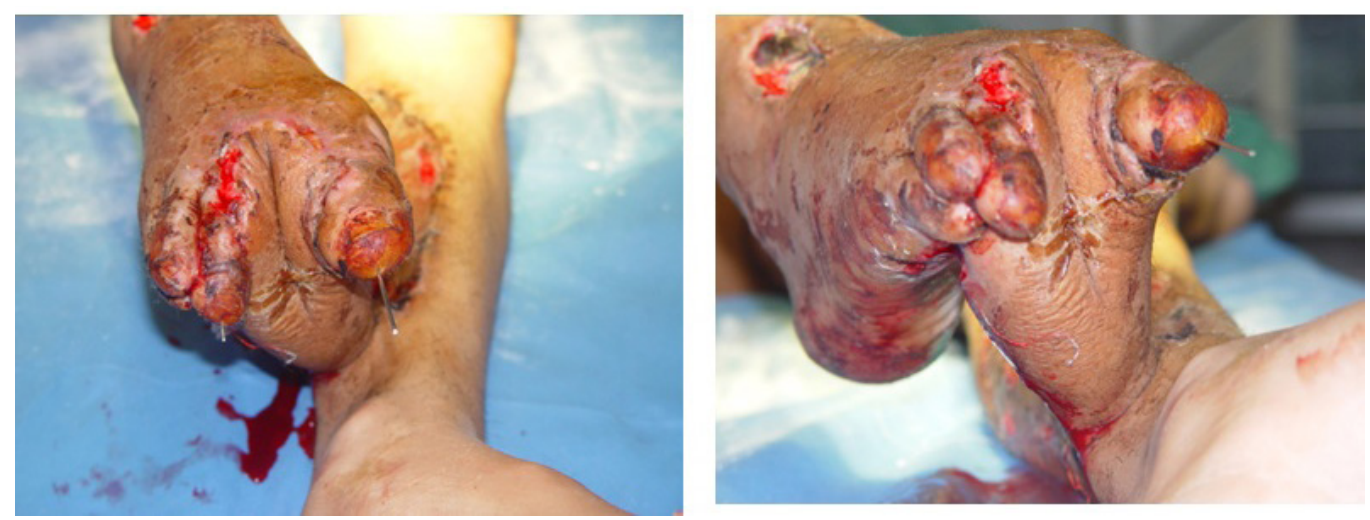

Figure 3. Cross-leg-transferring repair.
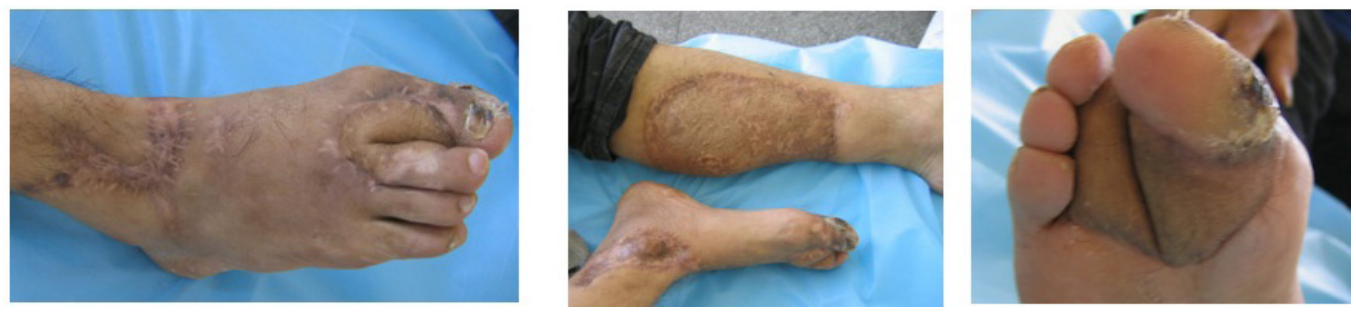

Figure 4. Seventeen months after repairing.

\section{DISCUSSION}

The repair of soft-tissue defects on the foot is always a difficult issue for a clinician because the foot is an important, exposed, and vulnerable part of the human body, being anatomically located at the distal aspect of the lower limb (Yildirim et al., 2002; Koshima et al., 2003; Chang et al., 2004). Before neurocutaneous vascular flaps were used, soft-tissue defects of the foot were usually repaired using local flaps, fascial flaps, free flaps, or reverse flow flaps, which are supplied and sustained by the major blood vessels of the lower limb. Local flaps and fascial flaps are suitable for repairing small-sized soft-tissue defects, whereas free flaps and reverse flow flaps, with the main blood vessels of lower legs as pedicles, can repair large-sized soft-tissue defects or wounds. However, when a soft-tissue defect on the foot is more extensive and the vascular supply network around the ankle is compromised, the application of a free flap and reverse flow flap is restricted.

The cross-leg neuro-fasciocutaneous flap is a traditional way of repairing a large-sized area of soft-tissue defects on the foot and lower limbs. This method involves 2 procedures. The first depends on a fascial flap and a random flap, with the pedicles located proximally. This procedure has the advantages of easily forming the flaps, generating reliable post-surgical outcomes, relying on low technology, having a short-operation time, and being used in clinical practice. Nevertheless, several disadvantages of the first flap procedure exist, such as insufficient incised flap area, limited physical positioning, and postural difficulties. The second 
flap procedure for cross-leg transfer mainly involves the use of reverse island flaps, which are supplied by a main artery of the contralateral leg. However, this surgical procedure is complex and time-consuming, involves repairing more extensive soft-tissue defects, and may result in a possible compromise of a main supplying artery to the contralateral leg. Thus, this procedure not only increases surgery risks, but also adds uncertainty to the subsequent survival and work capacity of patients.

Since Masquelet et al. (1992) re-introduced neurocutaneous vascular flaps to clinical practice, local and foreign researchers have successively reported several clinical studies on this type of flap (Geddes et al., 2003; Hallock, 2003; Zhang et al., 2004; Bhattacharya et al., 2005; Francesco, 2007). At present, pedicled perforator flaps have been the preferred method (Touam et al., 2001; Kneser et al., 2005; Chai et al., 2007; Dong et al., 2008a,b; Parrett et al., 2009) for repairing the lower extremities, especially soft-tissue defects on the ankle.

In this series cases that we have reported, the soft-tissue defects on the foot were all caused by electrical burns that have the following characteristics: wounds caused by foot injuries were large, and the bones, joints, tendons, nerves, and blood vessels were exposed; and, when electrical burns were observed around an ankle, the vascular supply network around that ankle suffers varying degrees of damage. The dorsal tibial arterial might vanish or weaken. In the above-mentioned case, free flap transposition, with vascular anastomosis, retrograde flap repair, and ligation of the ipsilateral anterior or posterior tibial vessels were all restricted. Therefore, the cross-leg flap transfer for the repair of soft-tissue defects in the contralateral leg becomes the only reliable choice for repairing a large-sized soft-tissue defect on the foot.

In this study, the terminal perforator of the posterior tibial and peroneal arteries was used as the flap rotation axis, with the cutaneous nerve, nutrient vessels, and deep fascia included in the pedicle in the cross-leg flap transfer for the repair of a large-sized soft-tissue defect of the foot. The cross-leg flap transfer has the following advantages: it is the only feasible method for repairing a large-sized soft-tissue defect in the foot when free flap and retrograde flap transfers cannot be performed; the flaps have sufficient length and pedicle size distally, which is convenient for repairing the affected limb; and the flaps have greater flexibility, which abates the perceived pain by the patients to a specific extent. Distally based pediculated perforator flaps formed using the sural nerve were used to repair the lower limb. The flaps accompanied the nutrient vessels and the greater saphenous vein as their vascular axis based on wound location. The flap can be provided with its own neurocutaneous vascular supply because incising the pedicle of the flaps includes the perforator vessels, deep fascia, and vascular axis. The area of the flap can be dissected nearly to the popliteal fossa, which extends from the antero-posterior to the midline aspect of the lower limbs. Thus, the requirements for repairing all types of softtissue defects on the foot are met. The flap dissection is very simple and does not involve a complex vascular anatomy, and will not compromise the main vessels of the lower limbs.

The main points to consider for the dissection of flaps are as follows: if larger flaps need to be dissected, a large-diameter perforator vessel should be selected using a Doppler ultrasound; all hardened and engorged veins in the pedicle should be carefully perforated and ligated after the formation of the flaps; and the width of the pedicle should have a diameter of about $5 \mathrm{~cm}$, such that the pedicle can be covered with multiple layers of Vaseline-treated gauze bandages, and are neither sutured with a leather tube nor require skin grafting for closure. The lower limb fixation should be stable and reliable.

The distally based neuro-fasciocutaneous flaps with blood vessel perforators are advantageous (Cavadas, 1997; Costa-Ferreira et al., 2001; Almeida et al., 2002; El-Shazly and 
Yassin, 2006; Afifi et al., 2008; Saint-Cyr et al., 2009) for lower limb reconstruction, especially for soft-tissue defects on the ankle and joints; however, necrosis of a part or most of the flaps usually occurs because of disturbances in venous drainage. To resolve this problem, several researchers have adopted the "anastomosed superficial venous method" in distally based neuro-fasciocutaneous flaps and designed interesting ways of transferring the flap to prevent pedicle distortion and compression. Moreover, several researchers have suggested that the superficial venous trunks should be preserved for the following reasons: first, the associated nutrient vessels and cutaneous nerve exist around the course of the superficial veins and, therefore, preserving these structures is advantageous to flap survival; second, ligating the superficial veins may damage the vascular supply network of the pedicle, which would affect the blood supply and venous drainage of the flaps.

Surgical methods that involve the anastomosis of superficial veins to the flap are ways of transferring the pedicle, which effectively maintains the venous drainage of the flaps and increases the flap survival rate. Nevertheless, the "anastomosed superficial vein method" requires vascular anastomosis, which is technically difficult to perform and has a prolonged operative time. Thus, these results imply that this method does not conform to the rule of "simple and effective" surgery. In this study, the anastomosed superficial vein hardened and engorged after the formation of the flaps, indicating that a communicating branch of a deep vein does not exist during anastomosis with the peripheral end of the superficial vein. Thus, venous blood reflux through the superficial vein cannot occur. However, the superficial vein trunk in the pedicle can be carefully separated and ligated, but no significant effect was noted on the flaps that formed. Moreover, the flap pedicle must be kept open instead of suturing with a leather tube or skin graft closure because this study dealt with pediculated perforator or neuro-fasciocutaneous flaps. Instead, we should only use multiple layers of Vaseline-treated gauze bandages to cover the pedicle for the following reasons: the edema in the distal pedicle is usually extensive, such that keeping the pedicle open is advantageous to relieve the disturbance in the venous drainage because of a compromised blood vessel supply network caused by the pedicular tissue edema. Some tissue fluid can be drained and venous pressure can be reduced because the pedicle is kept open.

\section{REFERENCES}

Afifi AM, Mahboub TA, Losee JE, Smith DM, et al. (2008). The reverse sural flap: modifications to improve efficacy in foot and ankle reconstruction. Ann. Plast. Surg. 61: 430-436.

Almeida MF, da Costa PR and Okawa RY (2002). Reverse-flow island sural flap. Plast. Reconstr. Surg. 109: 583-591.

Bhattacharya V, Deshpande SB, Watts RK, Reddy GR, et al. (2005). Measurement of perfusion pressure of perforators and its correlation with their internal diameter. Br. J. Plast. Surg. 58: 759-764.

Cavadas PC (1997). Reversed saphenous neurocutaneous island flap: clinical experience. Plast. Reconstr. Surg. 99: 19401946.

Chai Y, Zeng B, Zhang F, Kang Q, et al. (2007). Experience with the distally based sural neurofasciocutaneous flap supplied by the terminal perforator of peroneal vessels for ankle and foot reconstruction. Ann. Plast. Surg. 59: 526-531.

Chang SM, Zhang F, Yu GR, Hou CL, et al. (2004). Modified distally based peroneal artery perforator flap for reconstruction of foot and ankle. Microsurgery 24: 430-436.

Costa-Ferreira A, Reis J, Pinho C, Martins A, et al. (2001). The distally based island superficial sural artery flap: clinical experience with 36 flaps. Ann. Plast. Surg. 46: 308-313.

Dong ZG, Wei JW and Liu LH (2008a). Clinical application of sural neurofasciocutaneous flap carrying distally adiofascial flap. Chin. J. Reparative Reconstr. Surg. 22: 631-632.

Dong ZG, Liu LH and Zheng L (2008b). Reconstruction of foot and ankle defect with reversed sural neurofasciocutaneous flaps in children. Zhonghua Zheng. Xing. Wai Ke. Za Zhi. 24: 20-22. 
El-Shazly M and Yassin O (2006). Increasing the success rate of the reversed-flow fasciocutaneous island sural flap: a clinical experience in 26 cases. Ann. Plast. Surg. 57: 653-657.

Francesco G, Kolker D and Michael HR (2007). Modified reverse sural artery flap with improved venous outflow in lowerleg reconstruction. Ann. Plast. Surg. 59: 563-565.

Geddes CR, Morris SF and Neligan PC (2003). Perforator flaps: evolution, classification, and applications. Ann. Plast. Surg. 50: 90-99.

Hallock GG (2003). Direct and indirect perforator flaps: the history and the controversy. Plast. Reconstr. Surg. 111: 855865.

Kneser U, Bach AD, Polykandriotis E, Kopp J, et al. (2005). Delayed reverse sural flap for staged reconstruction of the foot and lower leg. Plast. Reconstr. Surg. 116: 1910-1917.

Koshima I, Itoh S, Nanba Y, Tsutsui T, et al. (2003). Medial and lateral malleolar perforator flaps for repair of defects around the ankle. Ann. Plast. Surg. 51: 579-583.

Masquelet AC, Romana MC and Wolf G (1992). Skin island flaps supplied by the vascular axis of the sensitive superficial nerves: anatomic study and clinical experience in the leg. Plast. Reconstr. Surg. 89: 1115-1121.

Parrett BM, Pribaz JJ, Matros E, Przylecki W, et al. (2009). Risk analysis for the reverse sural fasciocutaneous flap in distal leg reconstruction. Plast. Reconstr. Surg. 123: 1499-1504.

Saint-Cyr M, Schaverien MV and Rohrich RJ (2009). Perforator flaps: history, controversies, physiology, anatomy, and use in reconstruction. Plast. Reconstr. Surg. 123: 132e-145e.

Touam C, Rostoucher P, Bhatia A and Oberlin C (2001). Comparative study of two series of distally based fasciocutaneous flaps for coverage of the lower one-fourth of the leg, the ankle, and the foot. Plast. Reconstr. Surg. 107: 383-392.

Yildirim S, Akan M and Aköz T (2002). Soft-tissue reconstruction of the foot with distally based neurocutaneous flaps in diabetic patients. Ann. Plast. Surg. 48: 258-264.

Zhang SM, Xu DC and Gu Y (2004). Link-pattern neuro-veno-neurofasciocutaneous flap: Vascular basis and clinical application. Chin. J. Clin. Anatomy 22: 13-16. 